\section{Selecting Films for ESL}

By Inger Smith

While a film can certainly enhance an English as a Second Language (ESL) lesson, using it effectively isn't as simple as one might think. Getting a projector, screen and film to the same teach ar must complete in order to make the effort worthwile all. The most complex, time-consuming part of using film is

Consider film literacy. For most Canadians, seasoned film
and TV viewers, the complex language of the cinema poses few problems. We easily untangle the time distortions of the medium. We recognize flashbacks and dream sequences for what they are. Strange shooting angles, unusual lighting, distortions of scale, montage, collage, animation techniques mother tongue. The analogies and cultural symbols of western cinematography speak a language which we absorb automatically, to such a degree that we want our film fast, with so many jolts per minute, and if the screen doesn't deliver we turn However, ESL students come from varied cultures and many some of them, the language of film may present as many difhave to as does he how to "read" a film, as Robert Flaherty said he had to teach native Samoans to do. Flaherty's experiences while making Nanook of the North tell an ESL teacher much. While making hever seen film befored part of it for his Eskimo subjects who had ran to them and tried to touch them. When a frame contained only part of an arm, they assumed the arm itself had been cut off.
Depth perception also is learned. Studies in which Zambian and Holmes, 1964) revealed that the interpretation of perspective cues isn't automatic - just because something is smaller doesn't mean everyone will think it's further away. tional cues - color and tonal contrasts which diminish in intensity as they recede from the viewer's eye.
This is not to suggest that all ESL students are film illiterates, but rather that an ESL instructor must not assume a consistent to situations where explanations of cinematic technique are needed. Such sensitivity can be developed by watching a variety of foreign films and studying the differences in pacing, symbolism and lechnique. Or considering one's own difficulty with other visual languages - how many of us relate to
abstract painting or to the symbolism in the work of Norval abstract painting, or to the symb?
Morrisseau, the Ojibway painter?

No two people experience a film in the same way; nowhere is ways which we can oft an Est class. Perceplion is fllered in ways which we can often not predict. We can guess at some of
the filters - age, sex, intelligence, formal education level; the degree to which the viewer can identify with the people in the film depending on their skin color, body language, dress and rent emotional state affect what is perceived.

In our department we've used the NFB film, Steel Blues,

Inger Smith, B.A., is presently C.D.A. co-ordinator at the University of Alberta, Edmonton. She bas worked as an E.S.L. teacher in
University.

24 successfully for years. It's a realistic portrayal of the problems from a Chilean prison. Recently It showed it to a class contain. ing a large proportion of refugees from Eastern Europe, many ganda" allowed in the capitalist school system? They petitioned the head of the department to have it removed from the school. Tremendous material for class discussion, certainly, but no deacher would want to arouse such emolions in students who stead of inspiring them to learn English, this film turned these students off.

Violence in film can have a similar effect by causing students A case in roint is come from war-torn areas to relive traumas superb teaching tool in many ways, but the violent scenes with
which it tells ils story sometimes contradict the message of the Humor also demands careful selection because it's so easily
film especially for ESL, The Heatwave Lasted Four Days and Star are oblivious to this fact.

It's also important to remember that some students may simply not be accustomed to sitting quietly and paying atten-
tion to a film. Some of the first teachers to work in remote Eskimo communities reported that their students often gol headaches from the rapid eye movement and nightmares from he violence. A student may not like film at all, but be too polite wists will this further the cause of teaching ESL?

Film selection takes a great deal of sensitive previewing, as

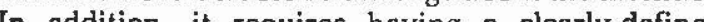
In addition, it requires having a clearly-defined teaching
objective and a plan for meeting these objectives. Some films can meet many objectives - stimulate discussion and research, give cultural information; act as starting points for practicin grajectives may require elementary film vocabulary. Som complex. Yet others may require that there be no dialogue at all - perhaps the objective is to allow students to create suitable

dialogue.
Of course, just watching a film isn't going to meet a lot of
objectives. After all, for many people film is an escape, a passive entertainment, not a teaching tool. Students may wonder how it relates to learning English. Indeed, it may not, unless ways have been provided for them to interact meaning fully with the Wy why they are walching a film and what they are expecise gain from the experience, that film's impact will be minimal. For this reason, 1 suggest planning film use in three stages.
preparation viewing and follow-up. See chart on next page for preparation, viewing and follow-up. (See chart on next page for follow - types of written or oral exercises, activitic ideas plays, discussions... I am always excited by the endless creativity a discussion of film use stimulates in workshops I do with ESL teachers. No other teaching aid does this to such a
degree. Therefore, in spite of the drawbacks, I can't help feelplexities involved (not to mention the hours of labor). But the challenge is an exciting one. For those who wish to accept it, eachers may wish to start a department film file to share viewing experiences and save viewing time. Secondly, the NFB leaching handbook, Teaching ESL with the Aid of Selected Films, discusses $60 \mathrm{NFB}$ films (20 in detail with exercises) which can

VOLUME 12, NUMBER 4, 1983
Title:

Lengt

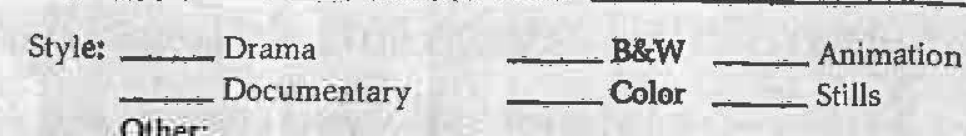

Language Level: __ Wilhout words A few words which Intermediate vocabular Advanced, complex vocabulary which must be understood to

Problems to Anticipate: Dated content Poot sound track / Poor quality print Too violent or otherwise offensive

Too long Other

What teaching objectives can Imeet by using this fin?

How can I use this film? PREPARATION

Teach grammar, idioms, vocabulary, gambits, conversational fillers which will be reviewed in he film, es,

Study, read, dramatize parts of dialogue or narration

Give out specific exercises about film which will direct students to look for specific answers Other

II. VIEWING EXERCISES

Take notes

Complete a partially written outline

ist specific types of items seen in film. ie, animals, verbs, etc

Turn off sound, create your own

Urm off sound, hold frame, ast questions, diseuss repeat word

Stop film, let students predict outcome

Cow fle only half the class who will explain it to others; then show it to others

Complete blanks in written narration

Oolit for answers to questions assigned in preparation

\section{FOLLOW-UP}

Make guestion for ofera bents about fil

Write summaries oulines paragens, compositions ist/classify things seen in film

-tell story (changing person, tense. I

"ye-witness". Describe a scene from memory. Write on board. Check as a group

Drescina

Discuss, debate different or opposing views in/about film

Createlact out skits

Other exercises: matching, filling-in blanks, sequencing, trueffalse, sentence completion, giving opinions, organizing ideas, interpreting chartstadditional information 